\title{
The design of the mooring system
}

\author{
Yating Liu ${ }^{1, a}$ \\ ${ }^{1}$ North China Electric Power University, Baoding, Hebei 071000,China; \\ a804269466@qq.com
}

\begin{abstract}
The design of the mooring system is to determine the length of chain type, quality and weight of the ball, the tilt angle of the buoy and draught of the swimming area and drums as small as possible. In this paper, the optimal design scheme of mooring system is presented based on mechanical analysis. Firstly, the mechanical model of mooring system is given, and the relationship between the variables in mooring system is found through the establishment of static equilibrium equations and geometric constraints. Then the iterative algorithm is used to solve the equation: Through a given depth, gradually solve each equation, the vertical depth can be calculated in the whole system, if the depth close to the given value, the iteration stops, otherwise continue the iteration. Using the iterative algorithm to repeat this process, we can obtain the draught of the buoy, drum tilt and swimming radius under different wind speed.
\end{abstract}

Keywords: iterative method, optimal design of mooring system.

\section{Mooring system model}

The transmission node of the near shallow sea observation network is composed of a buoy system, mooring system and underwater acoustic communication system ( Figure 1). The buoy system of a certain type of transmission node can be simplified into a cylinder. The mooring system is composed of a steel pipe, steel, heavy ball, welding anchor and special anti-drag anchor. It is required that the tangent direction of the end of the anchor chain and the anchor should not exceed 16 degrees, or the anchor will be towed, resulting in the loss of node displacement. Underwater acoustic communication system is installed in a sealed cylindrical drum. There are 4 steel drums, a welding anchor.

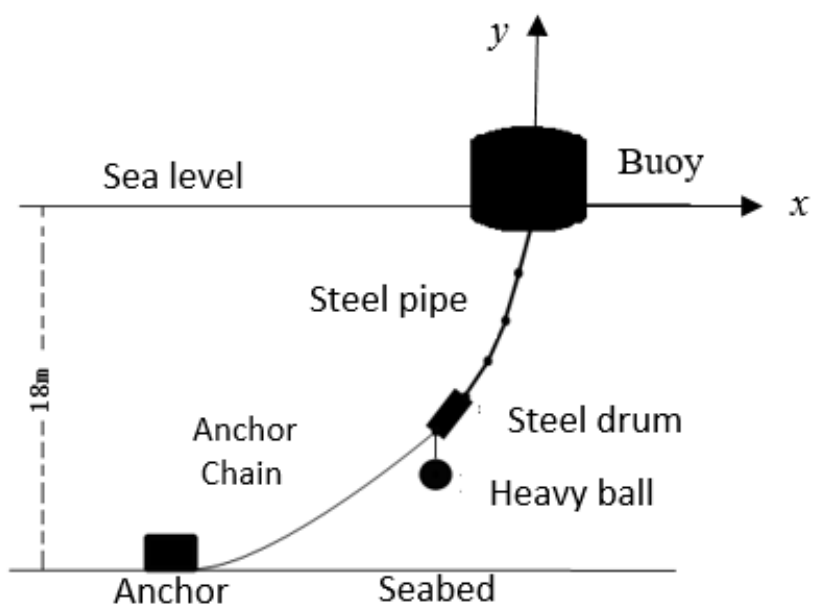

Fig. 1 Schematic diagram of the transmission node

\subsection{Model hypothesis}

(Only the schematic of the structure module, without considering the scale)

1) It is assumed that the wind speed and the water velocity along the water plane direction, and the plane flow, that is, there is no disturbance in the upper and lower, no component in the vertical direction.

2) If the anchor chain cannot bear the shear force, it can only bear the pulling force.

3) Suppose the seabed is an ideal plane. 
4) The weight of the ball and chain is too small so the buoyancy is negligible.

\subsection{Model hypothesis}

The relationship between the depth of sea water $\mathrm{H}$, the length $l_{i}$ and inclinations $\theta_{i}$ of each component satisfies the following conditions:

$$
H=h=\sum l_{i} \sin \theta_{i}+y_{0}
$$

We established the coordinate system as shown in the figure1, then the coordinates of each part of the mooring system are analyzed by taking the buoy as the coordinate origin.

\subsubsection{The force analysis of the buoy}

Set the direction of the wind speed is x positive direction. The force of the buoy has gravity G0, buoyancy F0, and the total wind load P, steel pipe 1 of the tensile force T1.

According to the Archimedes principle, the buoyancy of the buoy is determined by the volume of the liquid in which it is displaced. Assuming that the depth of the buoy is $\mathrm{Y} 0$, the buoyancy of the buoy is F0:

$$
F_{0}=\rho g V=\rho g \pi R^{2} y
$$

According to the offshore wind load calculation, wind load of P buoy satisfy the following formula:

$$
P=S v^{2}=\left(l-y_{0}\right) D v^{2}
$$

The tensile force of the 1 pairs of steel tubes on the buoy is T1, and the angle between the force and the $\mathrm{y}$ axis is $\theta 0$. According to the static balance principle, we have the following relations:

$$
\left\{\begin{array}{l}
T_{1} \cos \theta_{0}=G_{0}-F_{0} \\
T_{1} \sin \theta_{0}=P
\end{array}\right.
$$
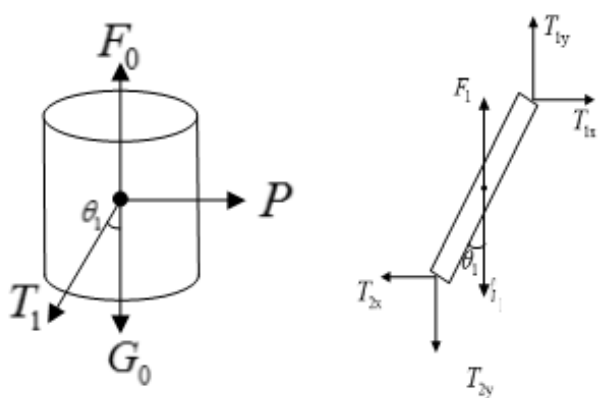

Fig. 2 Buoy Fig. 3 Steel pipe 1

\subsubsection{The force analysis of the buoy}

The steel pipe 1 is subjected to the force analysis, the steel tube is subjected to the gravity G1, the buoyancy F1, the buoy to the steel pipe 1 of the tensile force T1.Because the steel pipe and the buoy and a few steel pipes are hinged and the direction is unknown, which is divided into two orthogonal force:

$$
\left\{\begin{array}{l}
T_{1} x=T_{2 x} \\
T_{1 y}+F_{1}=T_{2 y}+G_{1}
\end{array}\right.
$$

The two force is expressed as orthogonal along the tube direction $T_{1 / \text { / }}$ and perpendicular to the pipe $T_{1 \perp}$ above. Assuming steel tilt is $\theta_{1}$, as shown below:

$$
\left\{\begin{array}{l}
T_{1 x} \cos \theta_{1}+T_{1 y} \sin \theta_{1}+\left(F_{1}-G_{1}\right) \cos \theta_{1}=T_{2 x} \cos \theta_{1}+T_{2 y} \sin \theta_{1} \\
T_{1 x} \sin \theta_{1}+T_{1 y} \cos \theta_{1}+\left(G_{1}-F_{1}\right) \sin \theta_{1}=T_{2 x} \sin \theta_{1}+T_{2 y} \cos \theta_{1}
\end{array}\right.
$$

As can be seen from the above formula, $\theta 1$ can be solved.

Similarly, the No.i steel pipe satisfies: 


$$
\left\{\begin{array}{l}
T_{i x} \cos \theta_{i}+T_{i y} \sin \theta_{i}+\left(F_{i}-G_{i}\right) \cos \theta_{i}=T_{i x} \cos \theta_{i}+T_{i y} \sin \theta_{i} \\
T_{i x} \sin \theta_{i}+T_{i y} \cos \theta_{i}+\left(G_{i}-F_{i}\right) \sin \theta_{i}=T_{i+1 x} \sin \theta_{i}+T_{i+1 y} \cos \theta_{i}
\end{array}\right.
$$

\subsubsection{The force analysis of the other part}

Similarly, the stress analysis of the steel drum satisfies:

$$
\left\{\begin{array}{l}
T_{5 / /}+\left(F_{5}-G_{5}-G_{m}\right) \cos \theta_{5}=T_{6 / /} \\
T_{5 \perp}+\left(G_{5}+G_{m}-F_{5}\right) \sin \theta_{5}=T_{6 \perp}
\end{array}\right.
$$

Next, we analyze the anchor chain. Ignoring the buoyancy of the anchor chain, assuming that the anchor cable cannot bear the shear force but only bear the tension, the anchor cable must be pulled along the direction of the anchor chain direction, as shown in the figure 5.

The length of the anchor chain is $L$, the length of each ring chain is $l$, then there is a $L / l$ section anchor chain, each chain has the force of gravity $\mathrm{G}$, tension $\mathrm{Ti}, \mathrm{Ti}+1$, by the plane force balance condition, we can obtain:

$$
\left\{\begin{array}{l}
T_{i} \cos \theta_{i}+G_{i}=T_{i+1} \cos \theta_{i+1} \\
T_{i} \sin \theta_{i}=T_{i+1} \sin \theta_{i+1}
\end{array} \quad(\mathrm{i}=6,7,8 \cdots)\right.
$$

Finally, the force of the anchor is analyzed. The force of the anchor is gravity, support force, and the tension of the last anchor chain TL/1+5:

$$
T_{L+l} \sin \theta \geq G
$$

\section{Iterative algorithm}

If we have known the depth of buoy draft, then the pulling force of steel pipe 1 buoy $\mathrm{T}$ size and direction can be solved by equation 1 . The second steel equation can also be solved .Then, each part of the system equation can be obtained. Verificatite $\mathrm{H}$, if the $\mathrm{H}$ is $18 \mathrm{~m}$, the result is reasonable draft, or modified draft again. Repeat the above process and iterative operation until the $\mathrm{H}$ is reasonable draught. Operation process can be shown as Fig.6: 


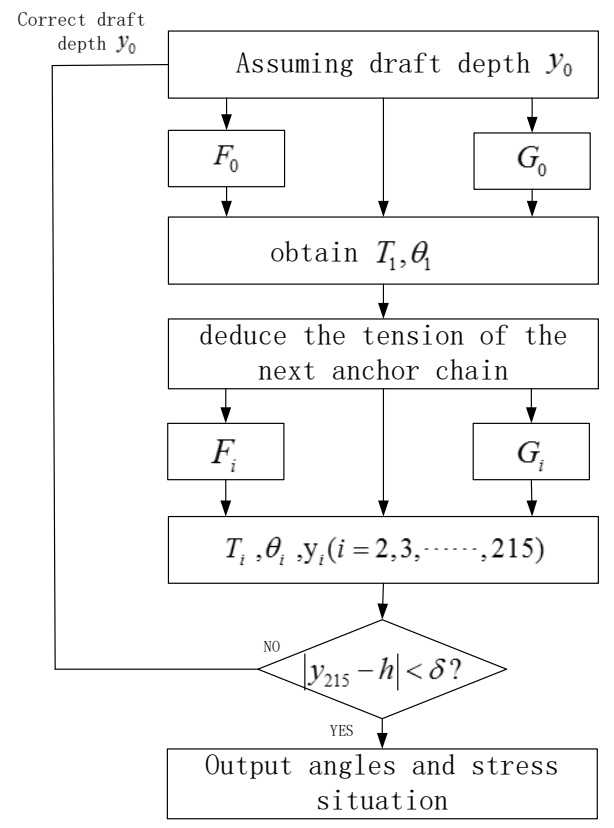

Fig.6 Iterative algorithm flow chart

Write a program and we can obtain the speed and steel joint pipes of the tilt angle, anchor shape, buoy and draught of the swimming area by iterative calculation.

\section{References}

[1]Gao Yue. Modified Algorithm of Sinusoid Signal Frequency Estimation Based on Quinn and Aboutanios Iterative Algorithms[A]. IEEE Beijing Section、IET Beijing Local Network、Beijing Jiaotong University.2016 IEEE 13th International Conference on Signal Processing Proceedings ( ICSP2016 ) [C].IEEE Beijing Section、 IET Beijing Local Network 、Beijing Jiaotong University:,2016:4.

[2]Cao Yalu,Peng Li,Li Jinzhou,Yang Le,Guo Fucheng. A new iterative algorithm for geolocating a known altitude target using TDOA and FDOA measurements in the presence of satellite location uncertainty[J]. Chinese Journal of Aeronautics,2015,(05):1510-1518.

[3]LI Shujun,WANG Hongguang,YANG Qiang. Constraint Force Analysis of Metamorphic Joints Based on the Augmented Assur Groups[J]. Chinese Journal of Mechanical Engineering,2015,(04):747-755.

[4]YANG Xue,WANG Hongbo,SUN Li,YU Hongnian. Operation and Force Analysis of the Guide Wire in a Minimally Invasive Vascular Interventional Surgery Robot System[J]. Chinese Journal of Mechanical Engineering,2015,(02):249-257.

[5]. An iterative algorithm for solving a class of matrix equations[J]. Journal of Control Theory and Applications,2009,(01):68-72. 